\title{
pH DE LA SUPERFICIE LUMINAL DE LA MUCOSA GASTROINTESTINAL DE CRÍAS DE ALPACAS DURANTE LAS PRIMERAS SEMANAS DE EDAD
}

\author{
PH of the Luminal Surface of the Gastrointestinal Mucosa of Baby \\ Alpacas During the First Weeks of Age
}

María Vásquez C. ${ }^{1,2}$, José Rodríguez G. ${ }^{1}$, Boris Lira M. ${ }^{1}$, Sergio Cueva M. ${ }^{1}$, Milder Ayón S. ${ }^{1}$, Yenny Mallma D. ${ }^{1}$

\section{RESUMEN}

\begin{abstract}
El objetivo del estudio fue determinar el pH de la superficie de la mucosa de los compartimentos estomacales y de los segmentos intestinales en los primeros 45 días de vida postnatal en la alpaca (Vicugna pacos). Se trabajó con 33 animales, procedentes de los departamentos de Puno y Cusco, desde el nacimiento (recién nacido - RN) hasta los 45 días de edad. Los animales fueron distribuidos en siete grupos etarios. El pH se determinó con un $\mathrm{pH}$ metro Metrohm 826 mobile con electrodo plano especial para membranas. Los animales fueron sacrificados y el tracto gastrointestinal fue delimitado en 13 porciones. En cada porción se hizo una apertura de $8 \mathrm{~cm}$ para determinar el pH de forma directa sobre la mucosa. El pH en los compartimentos 1, 2 y 3 porción abomaso fue menor en los RN ( $<<0.05)$, se incrementó en la primera semana de edad y se mantuvo constante hasta los 45 días de edad. El pH en las porciones intestinales fue constante en los grupos etarios, aunque se observó una ligera tendencia a la acidez en el intestino delgado y una tendencia a la neutralidad en el intestino grueso, lo que sugiere que las alpacas de estas edades tienen diversos mecanismos homeostáticos que mantienen constante el pH del microclima en todo el tracto gastrointestinal a pesar de los cambios en la alimentación durante su desarrollo inicial.
\end{abstract}

Palabras clave: $\mathrm{pH}$, mucosa gastrointestinal, alpaca

\section{Abstract}

The objective of this study was to determine the $\mathrm{pH}$ in the luminal mucosa of the gastric compartments and intestinal segments during the first 45 postnatal days of age in the alpaca (Vicugna pacos). Thirty three alpacas from birth to 45 days of age were distributed in seven age groups. The animals proceeded from the Puno and Cusco departments of Peru. The $\mathrm{pH}$ was measured using a $826 \mathrm{pH}$ mobile meter with a special

\footnotetext{
${ }^{1}$ Laboratorio de Fisiología Animal, Facultad de Medicina Veterinaria, Universidad Nacional Mayor de San Marcos, Lima

${ }^{2}$ E-mail: evasquezc@gmail.com
} 
flat electrode for membranes. Animals were slaughtered and the gastrointestinal tract was divided in 13 segments. An 8-cm length opening was done in each segment and $\mathrm{pH}$ was directly measured over the mucosa. The $\mathrm{pH}$ of compartments 1,2 and 3 (segment abomasum) was lower at birth $(\mathrm{p}<0.05)$, increased during the first week and kept constant until 45 days of age. The $\mathrm{pH}$ in the intestinal segments was constant in all age groups, but there was a slight tendency to acidity in the small intestine and a tendency to neutrality in the large intestine. This suggests that alpacas of these ages have homeostatic mechanisms for maintaining constant the $\mathrm{pH}$ of the microclimate in the gastrointestinal tract despite the change of feeds during the first phase of postnatal life.

Key words: $\mathrm{pH}$, gastrointestinal mucosa, alpaca

\section{INTRODUCCIÓN}

Los camélidos sudamericanos presentan un sistema digestivo similar al de los rumiantes, pero presenta ciertas características fisiológicas que favorece la degradación de pasturas con alto contenido de fibra. Este sistema digestivo está compuesto por tres compartimentos estomacales: compartimento 1 (parecido al rumen), compartimento 2 (parecido al retículo), y compartimento 3 (con una porción similar al omaso y una porción parecida al abomaso) (Vallenas et al., 1971); además del intestino delgado, ciego y colon (colon ascendente, colon transverso y colon descendente) (Sato y Montoya, 1989; Paredes, 2010).

El proceso digestivo y absorción de nutrientes en el tracto gastrointestinal se encuentra favorecido por el $\mathrm{pH}$ de la mucosa intestinal. El pH se encuentra en un amplio rango, es decir, entre 1.0 a 8.0, dependiendo del sector involucrado. El pH del estómago varía entre 1.0 a 3.0, en el intestino delgado de 5.0 a 7.0 y en el intestino grueso es alrededor de 8.0. El mantenimiento de estos valores influyen positivamente en la homeostasis celular, así como en la absorción de nutrientes y fármacos.

El pH de la mucosa gastrointestinal se debe la presencia de sustancias secretadas por los enterocitos y agentes microbianos residentes en el intestino (Castell y Moore,
1971), así como a la secreción de otros grupos celulares como las células caliciformes y las células de Paneth. Cambios en el $\mathrm{pH}$ extracelular cambian el pH intracelular de los enterocitos, que su vez modifican su función secretora y absortiva utilizando diversos mecanismos para mantener su equilibrio intracelular; principalmente, con la secreción de fluidos ricos en $\mathrm{H}^{+} \mathrm{y} \mathrm{HCO}_{3}^{-}$, regulando de esta manera el $\mathrm{pH}$ intracelular (Shimada y Hoshi, 1987; Hoinard y Gore, 1988; Wenzl et al., 1989).

En el caso del yeyuno, su acidificación inhibe la secreción ácida del estómago por un mecanismo neurohormonal mediado por somatostatina y colecistocinina (CCK); modificando, además, la secreción de glándulas anexas y la contractibilidad del tracto gastrointestinal (Orloff et al., 1992). El complejo mioeléctrico migratorio es gobernado por el pH duodenal (Woodtli y Owyang, 1995).

A nivel de colon, la alcalinización del pH favorece una eficiente absorción de electrolitos débiles como amoniaco y ácidos grasos de cadena corta (AGCC) (Castell y Moore, 1971). El descenso del pH en el colon humano (McNeil et al., 1987), en el ciego de la rata (Fleming et al., 1991) y en el colon del cuy (Oltmer y von Engelhardt, 1994) afecta los índices de absorción de AGCC, dado que la superficie luminal del intestino grueso requiere un microclima con $\mathrm{pH}$ constante (Feldman y Ickes Jr, 1997). El transporte de di y tripéptidos a través del PEPT1 (trans- 
portador de péptidos) en el intestino delgado está influenciado por el gradiente de protones y la acidificación del pH en el yeyuno (Daniel y Kottra, 2004), lo cual contribuye al transporte de nutrientes en el organismo (Chen et al., 2010). También se sabe que el aumento en el $\mathrm{pH}$ intestinal por encima de 7.0 cambia la afinidad de los transportadores de sodio y fosfato, disminuyendo su absorción a nivel intestinal cuando llega a 7.6 (Danisi et al., 1984).

Alteraciones del pH luminal en el tracto intestinal favorecen la presentación de enfermedades; así se tiene que la elevación del pH en el estómago y región pilórica del duodeno favorece el desarrollo de la infección con Helicobacter pylori e induce mecanismos de apoptosis en el epitelio (Suzuki et al., 2002). Asimismo, la acidificación de la mucosa es una característica de la sepsis y está relacionada con cuadros de colitis ulcerativa que inducen la producción de óxido nítrico, lo cual incrementa la permeabilidad epitelial por daño del citoesqueleto mediado por oxidantes y depleción de ATP (Unno et al., 1997).

Estudios en ratas y aves infectadas con Trichinella zimbabwensis han demostrado que el aumento del $\mathrm{pH}$ y osmolaridad del intestino facilitan el establecimiento y supervivencia del parásito (Tortora y Grabowski, 2003; Magwedere y Mukaratirwa, 2008). La infección con Escherichia coli está relacionada con la acidificación del intestino a través de la participación de uroguanilina y guanilina, que son ligandos exógenos para la guanilil ciclasa acoplada a membrana y que sirve como receptor para la enterotoxina de la bacteria, induciendo diarrea de tipo secretor (Vaandrager, 2002); estas dos proteínas son más efectivas en regiones ácidas del intestino delgado donde estimula la secreción de $\mathrm{HCO}^{-} \mathrm{y} \mathrm{Cl}^{-}$vía transportadores de membrana (Joo et al., 1988). La presentación de cuadros diarreicos causados por E. coli, Clostridium sp y parásitos en crías de alpacas (Vicugna pacos) es frecuente (Rivera, 1990), y se ha podido determinar que la mayoría de infecciones con E. coli están rela- cionadas a un $\mathrm{pH}$ fecal entre 7.5 y 9.0, siendo el $\mathrm{pH}$ fecal normal entre 7.0 y 8.1.

No existen estudios sobre $\mathrm{pH}$ gastrointestinal normal y su influencia en cuadros de infecciones entéricas en camélidos sudamericanos. Por tal motivo, el presente estudio tuvo por objetivo determinar el $\mathrm{pH}$ luminal de varias porciones gastrointestinales en crías de alpacas.

\section{Materiales y Métodos}

\section{Lugar de Estudio y Animales}

El estudio se llevó a cabo con alpacas provenientes de comunidades de los departamentos de Puno y Cusco, por encima de los $3500 \mathrm{msnm}$. El pH intestinal se determinó en la Estación Experimental del Centro de Investigación IVITA-Maranganí, y el procesamiento de datos se realizó en el Laboratorio de Fisiología Animal, Facultad de Medicina Veterinaria, Universidad Nacional Mayor de San Marcos, Lima.

Se emplearon 33 crías de alpaca, desde recién nacidas (RN) a 45 días de edad, distribuidas en siete grupos etarios: RN, 1-5, 6-11, 12-21, 27-31, 32-37 y 39-45 días de edad. Los animales utilizados en el presente estudio eran animales que los comuneros los habían destinado para el consumo humano. En el manejo y posterior sacrificio de los animales se siguió los "Principios Directrices Internacionales para la Investigación Biomédica que implique el Uso de Animales" del Consejo de Organizaciones Internacionales de las Ciencias Médicas (CIOMS, 1985).

\section{Determinación de pH Intestinal}

Inmediatamente posterior al sacrificio, se ingresó a la cavidad abdominal y se expuso las vísceras abdominales, removiendo el intestino delgado y grueso. Se hizo una incisión longitudinal de 5 a 8 cm en cada porción intestinal hasta observar la mucosa, para la determinación del $\mathrm{pH}$. 
El pH se determinó con un potenciómetro Metrohm 826 mobile, calibrado con la solución estándar del instrumento. La determinación de $\mathrm{pH}$ se realizó de forma directa, colocando el electrodo de membrana plana sobre la mucosa. Para esto, el tracto gastrointestinal se dividió en: compartimento 1 dorsal (C1 dorsal), compartimento 1 ventral (C1 ventral), compartimento 2 (C2), compartimento 3 omaso (C3 omaso), compartimento 3 abomaso (C3 abomaso), duodeno, yeyuno, íleon, ciego, colon ascendente (CA), colon transverso (CT), colon descendente (CD) y recto.

Los valores de $\mathrm{pH}$ intestinales fueron expresados como promedio. La comparación de los valores entre las porciones del tracto gastrointestinal se hizo por medio de un análisis de varianza de una sola vía.

\section{Resultados y Discusión}

El pH más bajo en los compartimentos 1, 2 y 3 porción omaso se presentó en el grupo etario de recién nacidos, para luego aumentar en la primera semana de edad $(p<0.05)$ y mantenerse constante en los grupos de edades mayores. En el caso del C3 abomaso, el $\mathrm{pH}$ fue ligeramente superior en el recién nacido, se incrementó durante la primera semana de edad, para ir descendiendo conforme aumenta la edad. El $\mathrm{pH}$ en esta sección fue estadísticamente mayor $(\mathrm{p}<0.05)$ en los primeros días de edad en comparación a los valores de $\mathrm{pH}$ a partir de grupo etario de 27-31 días de edad (Cuadro 1).

Los valores de $\mathrm{pH}$ en los segmentos intestinales fueron constantes en los siete grupos etarios (Cuadro 2). No obstante, se pudo determinar una ligera acidez en el intestino delgado (duodeno, yeyuno e íleon), que fue desde $6.24 \pm 0.30$ a $6.91 \pm 0.33$, mientras que a nivel del intestino grueso (ciego, colon y recto) hubo tendencia a la neutralidad (6.52 \pm 0.52 a $7.19 \pm 0.17$ ). La existencia de un $\mathrm{pH}$ constante del microclima en la superficie luminal de la mucosa del intestino ha sido demostrada en otros estudios, empleando microelectrodos sensibles a pequeños cambios del pH (Rechkemmer et al., 1986; McNeil, 1988).

Se ha demostrado que el $\mathrm{pH}$ del microclima de la superficie del intestino delgado es el resultado de un equilibrio dinámico entre la secreción y la absorción de H+ a través de la membrana luminal (en condiciones normales la secreción de $\mathrm{H}+$ se considera predominante sobre su absorción) y la difusión de fluidos mucosos hacia la luz intestinal, los que contienen bases como el $\mathrm{OH}^{-}$o $\mathrm{HCO}^{-}$(Blair et al., 1975).

La tendencia no significativa a la acidez a nivel del yeyuno en comparación con el íleon (Cuadro 2) fue confirmada por Hubel (1969), quien demostró que los dipéptidos y aminoácidos neutros se absorben mejor a nivel de yeyuno, ya que necesitan una mayor acidez para su absorción por usar un cotransportador que permite el ingreso de dipéptidos ante la mayor presencia de $\mathrm{H}+$ (Ganapathy y Leibach, 1983; Ganapathy et al., 1984; Takuwa et al., 1985). El pH de esta región tiene que mantenerse relativamente constante, pues tiene una influencia decisiva en la absorción de electrolitos débiles y de ácidos grasos de cadena corta (AGCC) (Rechkemmer, 1991).

Por otro lado, estudios en yeyuno de diversas especies animales han demostrado que para que se mantenga el $\mathrm{pH}$ estable se requiere tener constante las concentraciones de $\mathrm{H}+$; sin embargo, estos niveles pueden sufrir modificaciones importantes en su concentración; por ejemplo, cuando se suprime el sodio en la dieta, el antiportador $\mathrm{Na}^{+}-\mathrm{H}^{+}$ no permite la salida a la luz del $\mathrm{H}+$ ocurriendo un aumento del $\mathrm{pH}$ intestinal (Murer et al., 1976; Gunther y Wright, 1983; Cassano et al., 1984); pero momentos después de la supresión de $\mathrm{Na}+\mathrm{y}$ del aumento del $\mathrm{pH}$, este último comienza a disminuir y alcanzar sus valores normales como producto de otros mecanismos que secretan $\mathrm{H}+$ a la luz intestinal (Kinne-Saffra, 1985). 
Cuadro 1. Valores de $\mathrm{pH}$ en las porciones gástricas en crias de alpaca de 0 a 45 días de edad

\begin{tabular}{ccccccc}
\hline $\begin{array}{c}\text { Edad } \\
\text { (dias) }\end{array}$ & $\begin{array}{c}\mathrm{N}^{\circ} \text { de } \\
\text { animales }\end{array}$ & $\begin{array}{c}\mathrm{C} 1 \\
\text { dorsal }\end{array}$ & $\begin{array}{c}\mathrm{C} 1 \\
\text { ventral }\end{array}$ & $\mathrm{C} 2$ & $\begin{array}{c}\mathrm{C} 3 \\
\text { Oma so }\end{array}$ & $\begin{array}{c}\mathrm{C} 3 \\
\text { Abomaso }\end{array}$ \\
\hline $\mathrm{RN}$ & 2 & $3.93^{\mathrm{a}}$ & $5.81^{\mathrm{a}}$ & $4.66^{\mathrm{a}}$ & $3.70^{\mathrm{a}}$ & $3.96^{\mathrm{ab}}$ \\
$1-5$ & 5 & $6.91^{\mathrm{b}}$ & $6.77^{\mathrm{b}}$ & $6.39^{\mathrm{b}}$ & $5.56^{\mathrm{a}}$ & $5.35^{\mathrm{a}}$ \\
$6-11$ & 5 & $6.29^{\mathrm{b}}$ & $6.36^{\mathrm{ab}}$ & $6.53^{\mathrm{b}}$ & $4.70^{\mathrm{a}}$ & $4.53^{\mathrm{ab}}$ \\
$12-21$ & 4 & $6.41^{\mathrm{b}}$ & $6.26^{\mathrm{ab}}$ & $6.43^{\mathrm{b}}$ & $4.20^{\mathrm{a}}$ & $4.05^{\mathrm{ab}}$ \\
$27-31$ & 3 & $6.16^{\mathrm{b}}$ & $6.20^{\mathrm{ab}}$ & $6.47^{\mathrm{b}}$ & $5.79^{\mathrm{a}}$ & $2.99^{\mathrm{b}}$ \\
$32-37$ & 5 & $6.28^{\mathrm{b}}$ & $6.38^{\mathrm{ab}}$ & $6.50^{\mathrm{b}}$ & $5.55^{\mathrm{a}}$ & $3.11^{\mathrm{b}}$ \\
$39-45$ & 5 & $5.95^{\mathrm{b}}$ & $6.03^{\mathrm{b}}$ & $6.36^{\mathrm{b}}$ & $5.59^{\mathrm{a}}$ & $3.22^{\mathrm{b}}$ \\
\hline
\end{tabular}

C. Compartimento; RN: recién nacido

${ }^{\mathrm{ab}}$ Superíndices diferentes indican diferencia estadística $(\rho<0.05)$

Cuadro 2. Valores de pH inte tinal en crías de alpaca de 0 a 45 días de edad

\begin{tabular}{cccccccccc}
\hline $\begin{array}{c}\text { Edad } \\
\text { (Días) }\end{array}$ & $\begin{array}{c}N^{\circ} \text { de } \\
\text { animales }\end{array}$ & Duodeno & Yeyuno & Íleon & Ciego & CA & CT & CD & Recto \\
\hline $\mathrm{RN}$ & 2 & 6.37 & 6.60 & 7.30 & 7.05 & 6.90 & 7.25 & 7.10 & 7.20 \\
$1-5$ & 5 & 6.49 & 6.64 & 7.18 & 6.49 & 6.61 & 6.95 & 6.77 & 6.97 \\
$6-11$ & 5 & 6.54 & 6.75 & 6.87 & 6.42 & 6.62 & 6.80 & 6.85 & 6.90 \\
$12-21$ & 4 & 6.36 & 6.48 & 6.94 & 6.64 & 6.93 & 6.93 & 6.86 & 6.90 \\
$27-31$ & 3 & 6.32 & 5.66 & 7.01 & 7.10 & 6.85 & 7.29 & 7.25 & 7.18 \\
$32-37$ & 5 & 6.07 & 6.64 & 6.84 & 7.19 & 7.07 & 7.04 & 7.05 & 6.98 \\
$39-45$ & 5 & 6.41 & 6.71 & 6.63 & 6.88 & 6.82 & 7.12 & 7.11 & 7.04
\end{tabular}

$\mathrm{RN}$ : recién nacido; $\mathrm{CA}$ : colon ascenderte; $\mathrm{CT}$ : colon transverso; $\mathrm{CD}$ : colon descendente

\section{Conclusiones}

- $\quad \mathrm{El} \mathrm{pH}$ en los compartimentos 1, 2 y 3 porción abomaso es menor en los recién nacidos que en animales entre el día 1 y 45 de edad $(\mathrm{p}<0.05)$.

- Los valores de $\mathrm{pH}$ intestinal entre el nacimiento y los 45 días de edad fueron constantes.

\section{Agradecimientos}

Los autores desean expresar su agradecimiento al personal de la Estación Experimental IVITA- Maranganí, por el apoyo brindado en el desarrollo del presente trabajo. El estudio fue posible gracias al financiamiento del Proyecto PIBAP No 68 del FINCYTCONCYTEC. 


\section{LiTERATURA CitadA}

1. Blair JA, Lucas ML, Matty AJ. 1975. Acidification in the rat proximal jejunum. J Physiol 245: 333-350.

2. Cassano G Stieger B, Murer H. 1984. $\mathrm{Na} / \mathrm{H}-$ and $\mathrm{Cl} / \mathrm{OH}$ - exchange in rat jejunal and rat proximal tubular brush border membrane vesicles. Studies with acridine orange. Pflugers Arch 400: 309317.

3. Castell DO, Moore EW. 1971. Ammonia absorption from the human colon. The role of nonionic diffusion. Gastroenterology 60: 33-42.

4. Chen $M$, Singh A, Xiao F, Dringenberg U, Wang J, Engelhardt $R$, Yeruva S, et al. 2010. Gene ablation for PEPT1 in mice abolishes the effects of dipeptides on small intestinal fluid absorption, short-circuit current, and intracellular pH. Am J Physiol Gastr L 299: G265-G274.

5. [CIOMS] Council for International Organizations of Medical Sciences. 1985. International Guiding Principles for Biomedical Research Involving Animals. [Internet]. Available in: http:// www.cioms.ch/publications/guidelines/ 1985_texts_of_guidelines.htm

6. Daniel H, Kottra G. 2004. The proton oligopeptide cotransporter family SLC15 in physiology and pharmacology. Pflugers Arch 447: 610-618.

7. Danisi G, Murer H, Straub RW. 1984. Effect of $\mathrm{pH}$ on phosphate transport into intestinal brush-border membrane vesicles. Am J Physiol 246: G180-G186.

8. Feldman GM, Ickes JW Jr. 1997. Net $\mathrm{H}+$ and $\mathrm{K}+$ fluxes across the apical surface of rat distal colon. Am Physiol 272: G54-G62.

9. Fleming SE, Choi SY, Fitch MD. 1991. Absorption of short-chain fatty acids from the rat cecum in vivo. J Nutr 121: 1787-1797.

10. Ganapathy V, Burckhardt G, Leibach FH. 1984. Characteristics of glycylsarcosine transport in rabbit intesti- nal brush-border membrane vesicles. J Biol Chem 259: 8954-8959.

11. Ganapathy V, Leibach FH. 1983. Role of $\mathrm{pH}$ gradient and membrane potential in dipeptide transport in intestinal and renal brush-border membrane vesicles from the rabbit. Studies with L-carnosine and glycyl-L-proline. J Biol Chem 258: 14189-14192.

12. Gunther RD, Wright EM. 1983. $\mathrm{Na}^{+}$, $\mathrm{Li}+$, and $\mathrm{Cl}$ - transport by brush border membranes from rabbit jejunum. J Membrane Biol 74: 85-94.

13. Hoinard C, Gore J. 1988. Cytoplasmic $\mathrm{pH}$ in isolated rat enterocytes. Role of $\mathrm{Na}+/ \mathrm{H}+$ exchanger. Biochim Biophys Acta 941: 111-118.

14. Hubel KA. 1969. Effect of luminal chloride concentration on bicarbonate secretion in rat ileum. Am J Physiol 217: 40-45.

15. Joo NS, London RM, Kim HD, Forte LR, Clarke LL. 1998. Regulation of intestinal Cl- and HCO3-secretion by uroguanylin. Am J Physiol 274: G633G644.

16. Kinne-Saffra E. 1985. Transport function of renal cell membrane: ATPdriven transport system. In: Kinne R (ed). Renal biochemistry: cells, membranes, molecules. Amsterdam: Elsevier. p 143174.

17. Magwedere K, Mukaratirwa S. 2008. Evaluation of intestinal $\mathrm{pH}$ and osmolality levels in rats (Rattus norvegicus) and chickens (Gallus gallus) experimentally infected with Trichinella zimbabwensis. Intern J Appl Res Vet Med 6: 166-174.

18. McNeil NI, Ling KL, Wager J. 1987. Mucosal surface $\mathrm{pH}$ of the large intestine of the rat and of normal and inflamed larged intestine in man. Gut 28: 707-713.

19. Munck BG Munck LK. 1999. Effects of $\mathrm{pH}$ changes on systems ASC and B in rabbit ileum. Am J Physiol 276: G173G184.

20. Murer H, Hopfer U, Kinne R. 1976. Sodium/proton antiport in brush-bordermembrane vesicles isolated from rat small intestine and kidney. Biochem J 154: 597-604. 
21. Oltmer S, Von Engelhard W. 1994. Absorption of short-chain fatty acids from the in-situ-erfused caecum and colon of the guinea pig. Scand $\mathrm{J}$ Gastroentero 29: 1009-1016.

22. Orloff SL, Bunnett NW, Walsh JH, Debas HT. 1992. Intestinal acid inhibits gastric acid secretion by neural and hormonal mechanisms in rats. Am J Physiol 262: G165-G170.

23. Paredes MP. 2010. Relación de medidas biométricas y el desarrollo macroscópico del intestino de la cría de alpaca (Vicugna pacos). Tesis de Médico Veterinario. Lima: Univ Nac Mayor de San Marcos. 56 p.

24. Rechkemmer G. 1991. The gastrointestinal system, transport of weak electrolytes. In: Handbook of physiology. New York: Oxford University Press. p 371-388.

25. Rechkemmer G, Wahl M, Kuschinsky $W$, von Engelhardt W. 1986. $\mathrm{pH}-$ microclimate at the luminal surface of the intestinal mucosa of guinea pig and rat. Pflugers Arch 407: 33-40.

26. Rivera C. 1990. Dinamica de excreción fecal de Escherichia coli enteropatógeno y detección de enterotoxina de Clostridium perfringes tipo A en alpacas al mes de vida. Tesis de Médico Veterinario. Ica: Univ Nac San Luis Gonzaga de Ica. 52 p.

27. Sato A, Montoya L. 1989. Anatomía macroscópica del aparato digestivo de la alpaca (Lama pacos). UNMSM Bol Téc $\mathrm{N}^{\circ} 6.20 \mathrm{p}$.

28. Shimada T, Hoshi T. 1987. Role of $\mathrm{Na}+/ \mathrm{H}+$ antiport in intracellular $\mathrm{pH}$ regulation by rabbit enterocytes. Biochim Biophys Acta 901: 265-272.
29. Suzuki H, Yanaka A, Shibahara T, Matsui H, Nakahara A, Tanaka N, Muto H, et al. 2002. Ammonia-induced apoptosis is accelerated at higher $\mathrm{pH}$ in gastric surface mucous cells. Am J Physiol-Gastr L 283: G986-G995.

30. Takuwa N, Shimada T, Matsumoto H, Himukai M, Hoshi T. 1985. Effect of hydrogen ion-gradient on carriermediated transport of glycylglycine across brush border membrane vesicles from rabbit small intestine. Jpn J Physiol 35: 629-642.

31. Tortora GJ, Grabowski SR. 2003. The digestive system. In: Principles of anatomy and physiology. Hoboken, NJ: John Wiley. p 331-340.

32. Unno N, Menconi MJ, Smith M, Aguirre DE, Fink MP. 1997. Hyperpermeability of intestinal epithelial monolayers is induced by NO: effect of low extracellular pH. Am J Physiol 272: G923-G934.

33. Vaandrager AB. 2002. Structure and function of the heat-stable enterotoxin receptor/guanylyl cyclase C. Mol Cell Biochem 230: 73-83.

34. Vallenas A, Cummings JF, Munnell JF. 1971. A gross study of the comparmentalized stomach of two new-world camelids, the llama and guanaco. J Morphol 134: 399-423.

35. Wenzl E, Sjaastad MD, Weintraub WH, Machen TE. 1989. Intracellular pH regulation in IEC-6 cells, a cryptlike intestinal cell line. Am J Physiol 257: G732-G740.

36. Woodtli W, Owyang C. 1995. Duodenal $\mathrm{pH}$ governs interdigestive motility in humans. Am J Physiol 268: G146-G152. 